\title{
Crosstalk between DnaA Protein, the Initiator of Escherichia coli Chromosomal Replication, and Acidic Phospholipids Present in Bacterial Membranes
}

Rahul Saxena $^{1, *}$, Nicholas Fingland ${ }^{2}$, Digvijay Patil ${ }^{1}$, Anjali K. Sharma ${ }^{1}$ and Elliott Crooke ${ }^{1,3}$

1 Department of Biochemistry and Molecular \& Cellular Biology Georgetown University Medical Center, Washington, DC 20007, USA; E-Mails: dap89@georgetown.edu (D.P.); anjali0623@gmail.com (A.K.S.); crooke@georgetown.edu (E.C.)

2 Jet Propulsion Laboratory, California Institute of Technology, M/S: 183-426, 4800 Oak Grove Drive, Pasadena, CA 91109, USA; E-Mail: nick.k.fingland@jpl.nasa.gov

3 Lombardi Comprehensive Cancer Center, Georgetown University Medical Center, Washington, DC 20007, USA

* Author to whom correspondence should be addresses; E-Mail: rs426@georgetown.edu; Tel.: +1-202-687-1642; Fax: +1-202-687-7186.

Received: 21 January 2013; in revised form: 3 April 2013 / Accepted: 6 April 2013 / Published: 17 April 2013

\begin{abstract}
Anionic (i.e., acidic) phospholipids such as phosphotidylglycerol (PG) and cardiolipin (CL), participate in several cellular functions. Here we review intriguing in vitro and in vivo evidence that suggest emergent roles for acidic phospholipids in regulating DnaA protein-mediated initiation of Escherichia coli chromosomal replication. In vitro acidic phospholipids in a fluid bilayer promote the conversion of inactive ADP-DnaA to replicatively proficient ATP-DnaA, yet both PG and CL also can inhibit the DNA-binding activity of DnaA protein. We discuss how cellular acidic phospholipids may positively and negatively influence the initiation activity of DnaA protein to help assure chromosomal replication occurs once, but only once, per cell-cycle. Fluorescence microscopy has revealed that PG and CL exist in domains located at the cell poles and mid-cell, and several studies link membrane curvature with sub-cellular localization of various integral and peripheral membrane proteins. E. coli DnaA itself is found at the cell membrane and forms helical structures along the longitudinal axis of the cell. We propose that there is cross-talk between acidic phospholipids in the bacterial membrane and DnaA protein as a means to help control the spatial and temporal regulation of chromosomal replication in bacteria.
\end{abstract}


Keywords: acidic phospholipids; DnaA protein; chromosomal replication; Escherichia coli

Abbreviations: Phosphatidylglycerol, PG; cardiolipin, CL; Escherichia coli, E. coli; phosphatidylethanolamine, PE; cytosine diphosphate diacylglycerol, CDP-DAG; phosphatidylserine synthase, PssA; phosphatidylserine, PS; phosphatidylglycerophosphate synthase, PgsA; phosphatidylglycerophosphate phosphatase, Pgp; cardiolipin synthase, ClsA; Bacillus subtilis, B. Subtilis; phospholipase D, PLD; origin of chromosomal replication, oriC; $10-N$-nonyl acridine orange, $N A O$; constitutively stable DNA replication, cSDR; 2'-(or-3')-O-( $N$-methylanthraniloyl) adenosine 5' tri-phosphate, MANT-ATP; origin recognition complex, ORC; pre-replication complex, pre-RC; regulatory inactivation of DnaA, RIDA; phosphatidylcholine, PC; DnaA reactivating sequences, DARS; 3-decynoyl- $N$-acetylcysteamine, DNAC; phosphatidylinositol, PI; monosialotetrahexosylganglioside, $\mathrm{GM}_{1}$; Phosphatadic acid, PA; Staphylococcus aureus, S. aureus .

\section{Introduction}

Escherichia coli inner membrane contains a mixture of phospholipids with a composition of approximately 70\% phosphatidylethanolamine (PE), 25\% phosphatidylglycerol (PG) and $\sim 5 \%$ cardiolipin (CL), with a small remaining fraction of metabolic intermediates [1]. The precursor of these phospholipid species is CDP-diacylglycerol (CDP-DAG). CDP-DAG can then be shunted through two pathways to make either zwitterionic PE or the acidic PG and CL (Figure 1). In one pathway, addition of serine to CDP-diacylglycerol via phosphatidylserine synthase (PssA) results in phosphatidylserine (PS), which subsequently get decarboxylated by phosphatidylserine decarboxylase to form PE [2-5]. Alternatively, transfer of glycerol-3-phosphate onto CDP-diacylglycerol, predominantely by phosphatidylglycerophosphate synthase (PgsA), followed by a subsequent dephosphorylation by phosphatidylglycerophosphate phosphatase (Pgp), leads to the synthesis of PG [3,5]. Two molecules of PG condense to form CL through the action of cardiolipin synthase (ClsA) [6-8].

Anionic phospholipids are ubiquitous in nature. For example, PG and CL are associated with the photosystem II complexes of higher plants [9-11]. CL has been shown to be present in bacterial membrane [12-14], mitochondrial inner membrane [14-17], and the hydrogenosome membrane of anaerobic protist and fungi [18]. Besides, serving as a component of membrane bilayer, acidic phospholipids appear to regulate several critical cellular functions via protein-lipid interactions governed by various mechanisms, such as ion-mediated salt bridges [19] and electrostatic interaction [20-23]. These functions include acidic phospholipid induced (particularly CL) apoptosis in mitochondria [24-26], oxidative phosphorylation [27,28], and regulation of respiratory complexes in bacteria [29,30] and yeast [31]. The interaction of CL with Lon protease, which is involved in degrading misfolded proteins [32,33] influences the action of Lon by inhibiting its proteolytic and ATPase activities [34].

In prokaryotes, the role of acidic phospholipids also appears to be linked to chromosomal and cell division-related events [35] including the initiation of chromosomal DNA replication [36-39]. In vivo evidence links proper cellular levels of PG and CL with continued cell growth [40,41] and normal chromosomal replication [36-39], in that reduced levels of acidic phospholipids, arising from 
repressed expression of $\operatorname{pgs} A$, result in arrested-growth and inhibited chromosomal replication in otherwise wild-type E. coli.

Figure 1. Biosynthesis of phospholipids in Escherichia coli. The synthesis of phospholipids is carried out in the steps as indicated and is catalyzed by the following enzymes that are encoded by the genes denoted at each step: (1) CDP-diacylglycerol synthase; (2) phosphatidylglycerolphosphate synthase; (3) phosphatidylglycerolphosphate phosphatase; (4) cardiolipin synthase; (5) phosphatidylserine synthase; (6) phosphatidylserine decarboxylase.

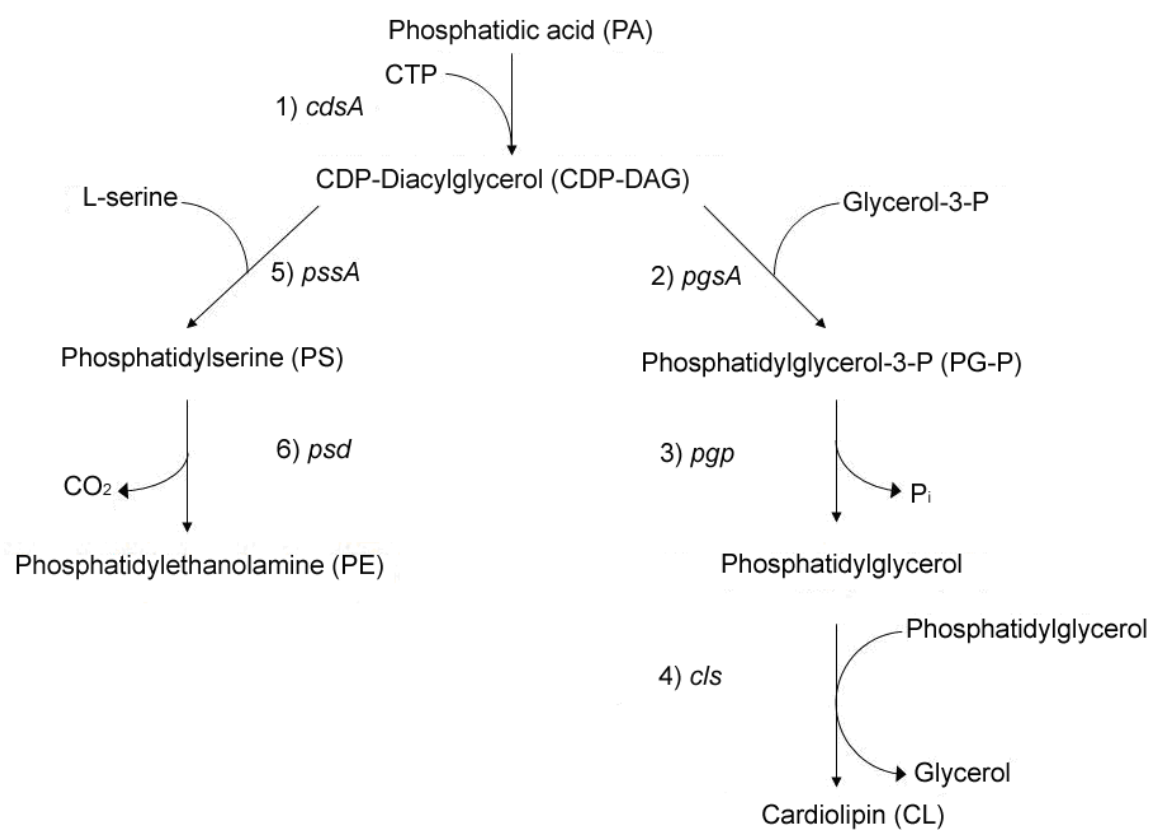

Growth of $c l s A$ mutants is affected to a lesser extent than that for $\operatorname{pgs}_{s} A$ mutants [40-42]. This in part may be due to the cells possessing redundant pathways for CL synthesis [7,43]. Bacillus subtilis mutants lacking the $c l s A$ gene still possess CL domains that appear after sporulation is initiated [44]. E. coli defective for ClsA activity also appear to maintain residual levels of CL, with PssA implicated in its formation possibly by donating a phosphatidyl group to glycerol [7]. Moreover, other genes homologous to $c l s$ have been identified in E. coli. One, named as $c l s B$ [45], also known as $f 413$ or $y b h O[43,45]$ encodes for a protein with the characteristic feature of having HKD motifs found in the phospholipase D (PLD) protein superfamily, which also includes cardiolipin synthase [45]. Biochemical characterization of the protein translated from E. coli clsB reveals that although kinetically less active, the protein can catalyze the formation of CL from PG. Thus, the possibility cannot be excluded that the $c l s B$ gene product can generate enough CL to support essential CL-dependent functions in clsA null cells. Moreover, a recent study has identified a third $c l s$ homologue, termed $c l s C$, which also contains HKD motifs [43]. Unlike the $c l s A$ gene product, the protein encoded by $c l s C$ uses $\mathrm{PE}$ as the phosphatidyl donor to $\mathrm{PG}$ for the formation of $\mathrm{CL}$, and does so in a manner dependent on coexpression of the $y m d B$ gene that precedes $c l s C$ in an operon. A triple cls $\mathrm{ABC}$ mutant has been shown to lack any detectable level of $\mathrm{CL}$ and has reduced viability when in the stationary phase [43].

A large body of in vitro and in vivo data indicates that the action of DnaA protein as the initiator of chromosomal replication is modulated by PG and CL residing in the fluid bilayer of the bacterial inner 
membrane [46-48]. Acidic phospholipids have the ability to promote the exchange of the tightly bound allosteric effectors ADP and ATP (see sections 3), and studies have shown that acidic phospholipids can inhibit the formation of the replicatively active nucleoprotein complex at the origin of chromosomal replication ( oriC) in $E$. coli [49,50] (see section 7). The molar ratios of membrane phospholipids appears to change as cells pass from exponential growth into stationary phase [51,52] and recent work shows that depletion of cellular acidic phospholipids leads to under initiation of replication from oriC during the cell-cycle [39].

Acidic phospholipids, particularly CL, are present in the form of lipid domains that can be visualized using the CL-specific fluorescent dye 10-N-nonyl acridine orange (NAO) [12,15]. These domains are localized at negatively-curved regions of bacterial cell membranes [53]. The role of acidic phospholipids in directing membrane curvature has been the focus of studies, as it has their role in the localization of various proteins, such as the cell division protein MinD [53-55], the osmosensory transporter, ProP [56-58] and the SecYEG protein complex [59,60]. With studies showing DnaA localized at the plasma membrane [61,62], the spatial arrangement of DnaA with respect to acidic phospholipid domains will be an interesting aspect to examine.

Based on acidic phospholipids affecting the nucleotide-bound state of DnaA, the ability of DnaA to productively bind to oriC, and the localization of DnaA at the cell membrane, we propose that there is cross-talk between the $E$. coli chromosomal initiator protein, DnaA, and acidic phospholipids present in the bacterial membrane. A review of supporting literature is presented below.

\section{Linkage between Bacterial Growth and Membrane Acidic Phospholipids}

Intriguing observations suggest that the total cellular anionic lipid content present in the membrane influences bacterial growth [40,41]. Growth of the bacterial cells harboring a sole chromosomally-encoded copy of the $\operatorname{pgs} A$ gene under an inducible promoter can be regulated by the absence or presence of the inducer in the medium [41]. When grown in the absence of the inducer, the cells continue to grow for several generations until the levels of PG and CL decrease to threshold amounts, at which point the cells undergo a growth-arrest. The arrested cells remain viable, and if $\operatorname{pgs} A$ expression is again induced, the cells resume growth shortly afterwards [41].

Interestingly, the growth-arrest of acidic phospholipid-depleted cells can be bypassed. One such mechanism is when bacterial cells can grow in the presence of otherwise insufficient levels of cellular acidic phospholipids because they possess mutations in $r n h A$ [36], which encodes for RNaseH that degrades RNA within RNA-DNA hybrids [63,64]. In contrast to normal DnaA protein-dependent replication initiation from oriC, these cells, in a RecA-dependent manner, use persistent RNA-DNA hybrids formed in absence of $\mathrm{RNaseH}$ to serve as sites for the initiation of DNA synthesis, a process termed constitutive stable DNA replication (cSDR) $[63,64]$.

In $E$. coli, the growth-arrested phenotype of a $g_{s} A$ deletion also can be reversed if the cells lack lpp, the gene encoding Lpp (murein lipoprotein), a major outer membrane lipoprotein [65]. Biosynthetic maturation and translocation of Lpp from the inner to outer bacterial membrane involves the transfer of the diacylglyceryl moiety from PG to cysteine-21 of prolipoprotein, producing the diacylglyceryl modified intermediate, DGPLP $[66,67]$. Blocking the diacylation of prolipoprotein by either lack of phosphatidylglycerol due to repressed $\operatorname{pgs}_{S} A$, or by introducing a cysteine-21 to glycine 
point mutation results in accumulation of unmodified, immature protein product (UPLP) in the inner cell membrane, coincident with reduced viability $[66,68]$. The defect in bacterial cell growth has been attributed to an anomalous covalent linkage between accumulated UPLP and peptidoglycan at the cell membrane [68]. However, another hypothesis yet to be addressed is whether accumulation of UPLP may also adversely affect oriC-dependent DNA initiation.

DnaA protein initiates chromosomal replication at oriC once per cell cycle. A third mechanism to suppress the growth arrest of acidic phospholipid-deficient cells, besides harboring the $r n h A$ genetic background that allows cSDR to occur, or cells having a lpp null mutation, is over-expression of DnaA protein possessing certain deletion and point mutations in its membrane-binding or DNA-binding domains (Figure 2) [37]. One well characterized mutant form of DnaA is DnaA(L366K), which can restore growth to acidic phospholipid-depleted cells [37,39].

Biochemically, DnaA(L366K) is similar to wild-type in several properties, including nucleotide binding and hydrolysis (see sections 2 and 3). Yet, DnaA(L366K) can initiate replication only when a limited amount of wild-type DnaA is present [69]. In agreement, nucleoprotein complexes (see section 2) containing only DnaA(L366K) protein were found inefficient at unwinding DNA duplex at oriC, and thus are unable to independently support DNA synthesis [70]. However, mixed oligomers containing DnaA(L366K) along with wild-type DnaA form productive nucleoprotein complexes [70]. The $N$-terminal domain of DnaA protein is responsible for oligomerization of DnaA-DnaA protomers (Figure 2) [71-73]. Therefore, mutations present either in the membrane binding domain or $C$-terminus DNA binding domain of DnaA protein might not affect formation of mixed, but functional heteroligomers between wild-type and the mutant forms of DnaA.

Figure 2. Schematic representation of DnaA protein domains. DnaA protein has four distinct functional domains. Domain I, comprising amino acid residues 1-86, and flexible linker region domain II (87-134) are involved in protein-protein interaction. Domain III (135-356) contains conserved features of the AAA + protein superfamily and is involved in ATP binding. The $C$-terminus of domain III features an amphipathic helix (357-374), which is responsible for DnaA binding to acidic membranes. Domain IV (375-467) is essential for DNA binding and nucleoprotein complex formation.

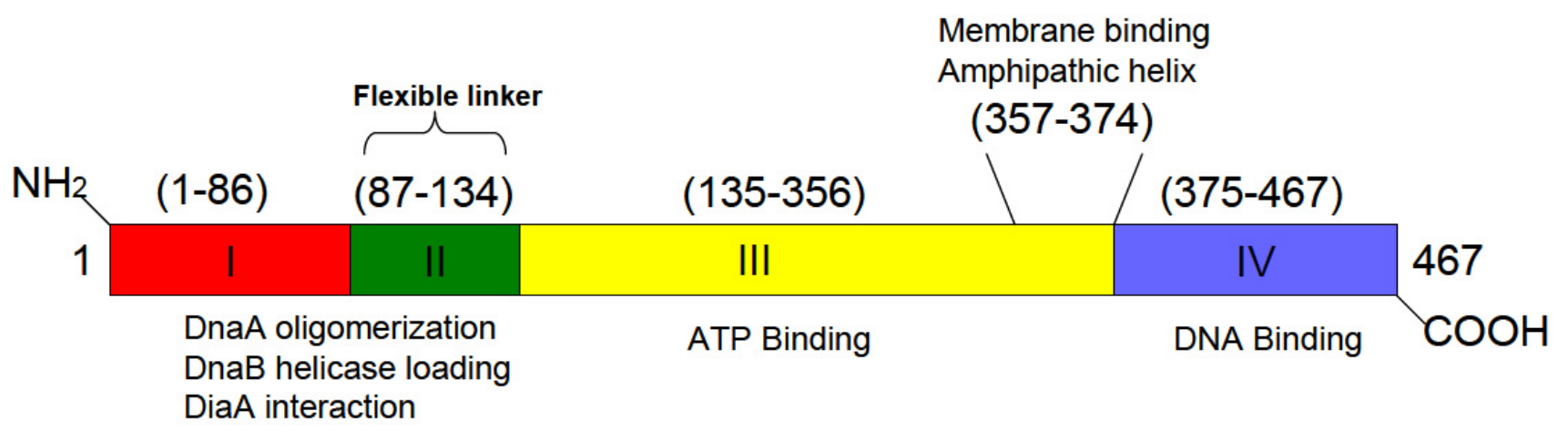

Limited structural data on DnaA protein in different bacteria provide a major challenge to understanding what conformational changes in DnaA(L366K) or mutant forms of DnaA with certain small, internal deletions in the $C$-terminal region allow over-expression of the mutant proteins, but not the wild type protein, to bypass the arrested-growth phenotype. However, findings from a study that 
used an ATP fluorescent analog, 2'-(or-3')-O-( $N$-methylanthraniloyl) adenosine 5 ' tri-phosphate (MANT-ATP), suggest that DnaA(L366K) might require a lower concentration of acidic phospholipids to induce the exchange of ADP to ATP bound to DnaA protein [74]. This study postulates that the low levels of $\mathrm{PG}$ and $\mathrm{CL}$ arising from repressed pgs $A$ expression may be sufficient for promoting ADP-ATP exchange on DnaA(L366K), but not wild-type DnaA.

\section{Importance of the Nucleotide State of DnaA Protein in Determining the Functional Status of Nucleoprotein Complex Generated at oriC}

For several decades DnaA has been known to be an essential protein involved in chromosomal replication [75-77]. Formation of a productive nucleoprotein complex of DnaA protein bound to oriC causes DNA conformational changes that trigger melting of nearby duplex DNA [77-79]. This is followed by DnaA-mediated recruitment of DnaB helicase to sites of the future replication forks at the melted double-stranded DNA, and ultimately the assembly of replisomes that will carry out bi-directional chromosomal replication [80-82].

In binding to the approximately 250 base pair region of oriC, several molecules of DnaA protein interact with multiple asymmetric DnaA-binding sequences, termed as R boxes [76,83], I sites [84], and $\tau$ sites [85]. DnaA tightly associates with the adenine nucleotides ATP and ADP $\left(K_{\mathrm{D}}\right.$ of 0.03 and $0.2 \mu \mathrm{M}$, respectively) [77]. However, whether ATP or ADP is tightly bound to DnaA protein, determines DnaA protein's preferential binding to specific oriC elements. The binding of ADP-DnaA or ATP-DnaA to R1, R2, and R4 boxes constitutes an origin recognition complex (ORC) (Figure 3) [70,86,87], which persists throughout most of the cell-cycle [88]. In contrast, low affinity I sites (I1, I2, I3 and I4) [70,84] and $\tau$ sites ( $\tau 1$ and $\tau 2$ ) [85] show preferential binding by only ATP-DnaA to generate a pre-replication complex (pre-RC) (Figure 3) [70,86,87]. The engagement of low affinity sites I2 and I3 with ATP-DnaA is required for the progression from an ORC to a pre-RC and DNA strand opening (Figure 3). Recently, it has been shown that at the time of initiation, DnaA protein extends the assembly from the high affinity to low affinity DnaA binding sites [87].

\section{Acidic Phospholipids Promote Conversion of Replicatively Feeble ADP-DnaA to the Replicatively Active ATP-Form}

One mechanism in $E$. coli to ensure that initiation occurs only once per cell-cycle is known as Regulatory Inactivation of DnaA (RIDA), which promotes the hydrolysis of DnaA-bound ATP, and thus the conversion of replicatively active ATP-DnaA to inactive ADP-DnaA, a process that involves Hda protein (homologous to DnaA) [89,90]. The hydrolysis of replicatively active ATP-DnaA to inactive ADP-DnaA is stimulated by the interaction of ATP-DnaA with ADP-Hda protein via inter-protein domain interactions [91,92]. It has been shown earlier that the cellular levels of ATP-DnaA are tightly controlled by Hda activity, as cells lacking hda gene, result in over-initiation of chromosomal replication [93,94]. Besides RIDA, hydrolysis of ATP-DnaA has also been attributed to another chromosomal locus, datA, previously described as reservoir for DnaA protein molecules, to prevent untimely initiation in a manner dependent on nucleoid-associated integration host factor (IHF) $[95,96]$. The resulting drop in the cellular concentration of ATP-DnaA, coupled with the synthesis of new DnaA-binding sites as genome duplication proceeds, lowers the initiation potential of 
DnaA protein below a needed threshold, and thereby preventing the re-initiation of replication during the same cell-cycle (Figure 4).

Figure 3. Schematic representation of oriC: Escherchia coli oriC (approximately 250 base pairs) contains cognate recognition sequences for DnaA protein. Based on their affinity for ADP-DnaA and ATP-DnaA these DNA elements are categorized as high affinity (R1, R2 and R4) and low affinity (R5M, I1, I2 I3, I4, tau 1 and tau 2 sites) DnaA binding sites. Binding of ADP-DnaA and ATP-DnaA to high affinity DnaA binding elements form an ORC. At the onset of chromosomal replication, the ORC is converted to a pre-RC by oligomerization of additional DnaA protein molecules to occupy low affinity DnaA binding sites.

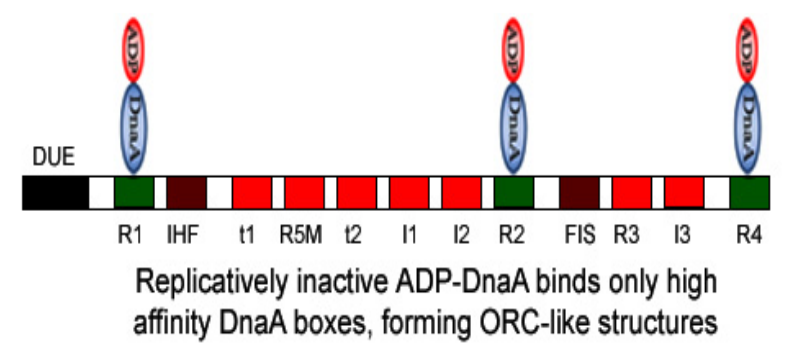

Replicatively inactive ADP-DnaA binds only high
affinity DnaA boxes, forming ORC-like structures

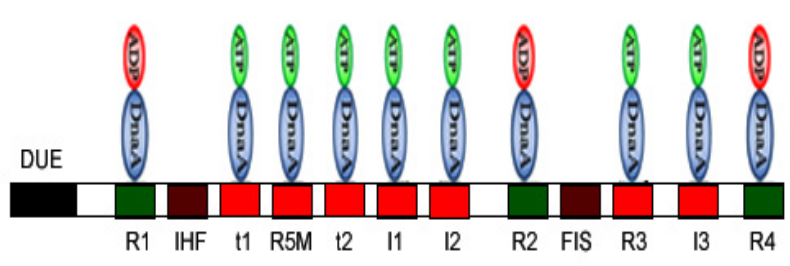

Replication proficient ATP-DnaA accumulates at additional cognate recognition sequences, converting bacterial ORC like structures to pre-replication complexes

DNA unwinding element ADP-DnaA High affinity DnaA binding boxes
bind both ATP-or ADP-DnaA

DNA sequences that bind architectural IHF and FIS

For the next round of chromosomal replication to occur in daughter cells, the initiation potential of DnaA must again rise above a certain threshold. Increased ATP-DnaA can occur through regeneration of ATP-DnaA from inactive ADP-DnaA in combination with de novo DnaA synthesis [97] (Figure 4). The in vitro exchange of bound ADP for ATP on purified DnaA is slow relative to the time of the bacteria cell cycle, with only half of purified ADP-DnaA converted to ATP-DnaA after 40 min, even in the presence of excess ATP [77]. However, there are mechanisms capable of accelerating the rejuvenation of ADP-DnaA to ATP-DnaA (Figure 4).

In vitro incubation of oriC-bound ADP-DnaA and excess ATP in the presence of acidic phospholipids, such as $\mathrm{PG}$ or $\mathrm{CL}$, results in rapid release of bound ADP and exchange for ATP [46-48]. CL is approximately 10-times more potent than other anionic lipids in promoting release of DnaA-bound nucleotide. In contrast, zwitterionic phospholipids, such as phosphatidylcholine (PC) and PE, fail to stimulate the release of DnaA-bound nucleotides [46,48,69]. Thus, acidic phospholipids have been proposed to catalyze the rejuvenation of ADP-DnaA to ATP-DnaA in vitro [46-48]. 
Figure 4. The cross talk between acidic phospholipids (APL) and DnaA. (A) Prior to initiation, inactive ADP-DnaA occupies high-affinity sites on oriC to form an ORC. As active ATP-DnaA concentration increases through acidic phospholipid stimulated DnaA exchange of ADP-ATP, DARS, and synthesis of new DnaA protein, low affinity DnaA binding sites in oriC are filled, and chromosomal replication is initiated; (B) After initiation, a combination of mechanisms to prevent re-initiation (i) RIDA (ii) sequestration of DnaA (iii) inhibition of DnaA binding to oriC by acidic phospholipid domains (iv) tritration of ATP-DnaA by other DnaA binding loci present at chromosome, such as datA, ensure initiation only occurs once per cell-cycle. Conversion of ADP-DnaA to ATP-DnaA may also occur through interaction with DARS.

A Initiation of chromosomal replication triggered by increased levels of ATP-DnaA from:

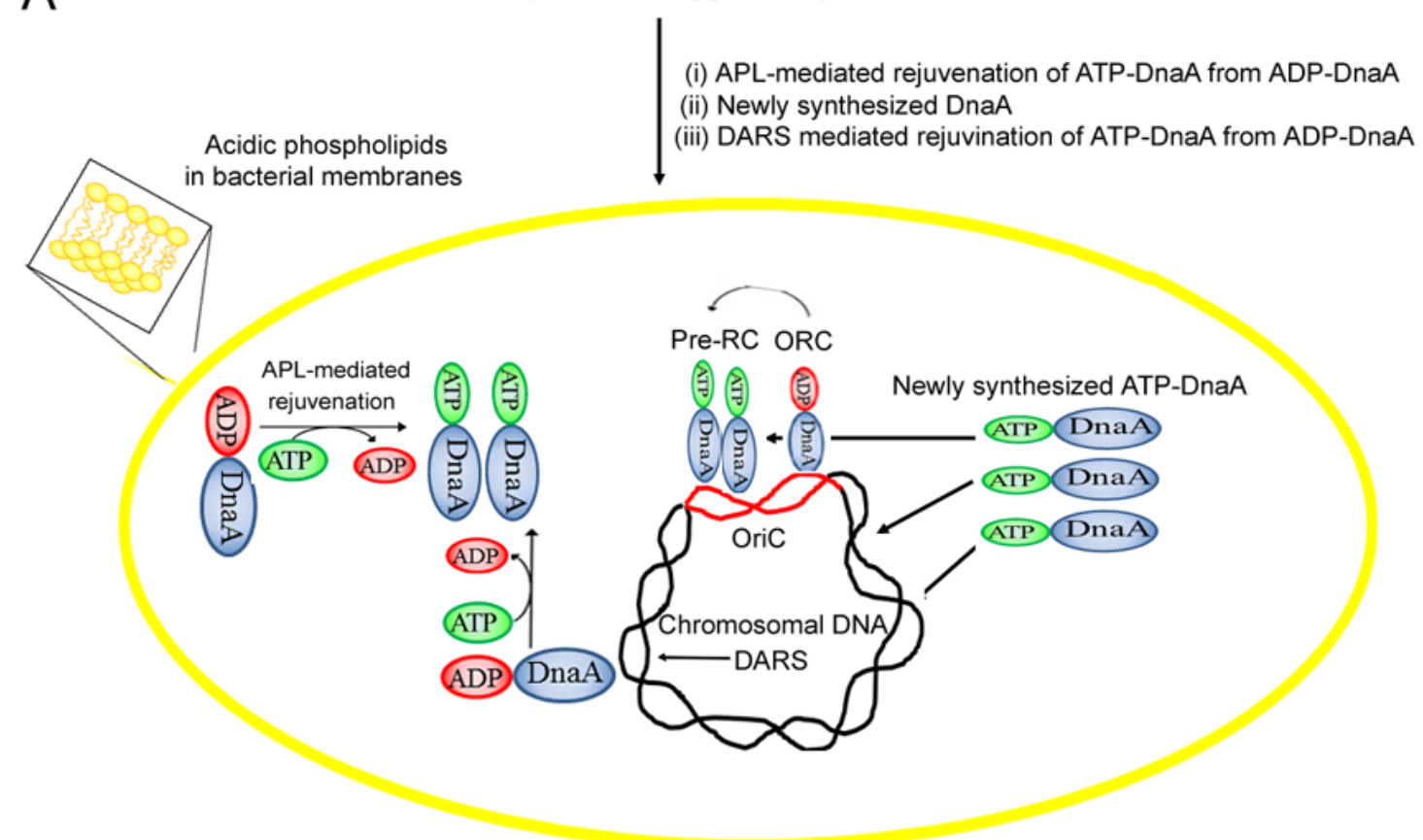

B Re-initiation of chromosomal replication prevented by:

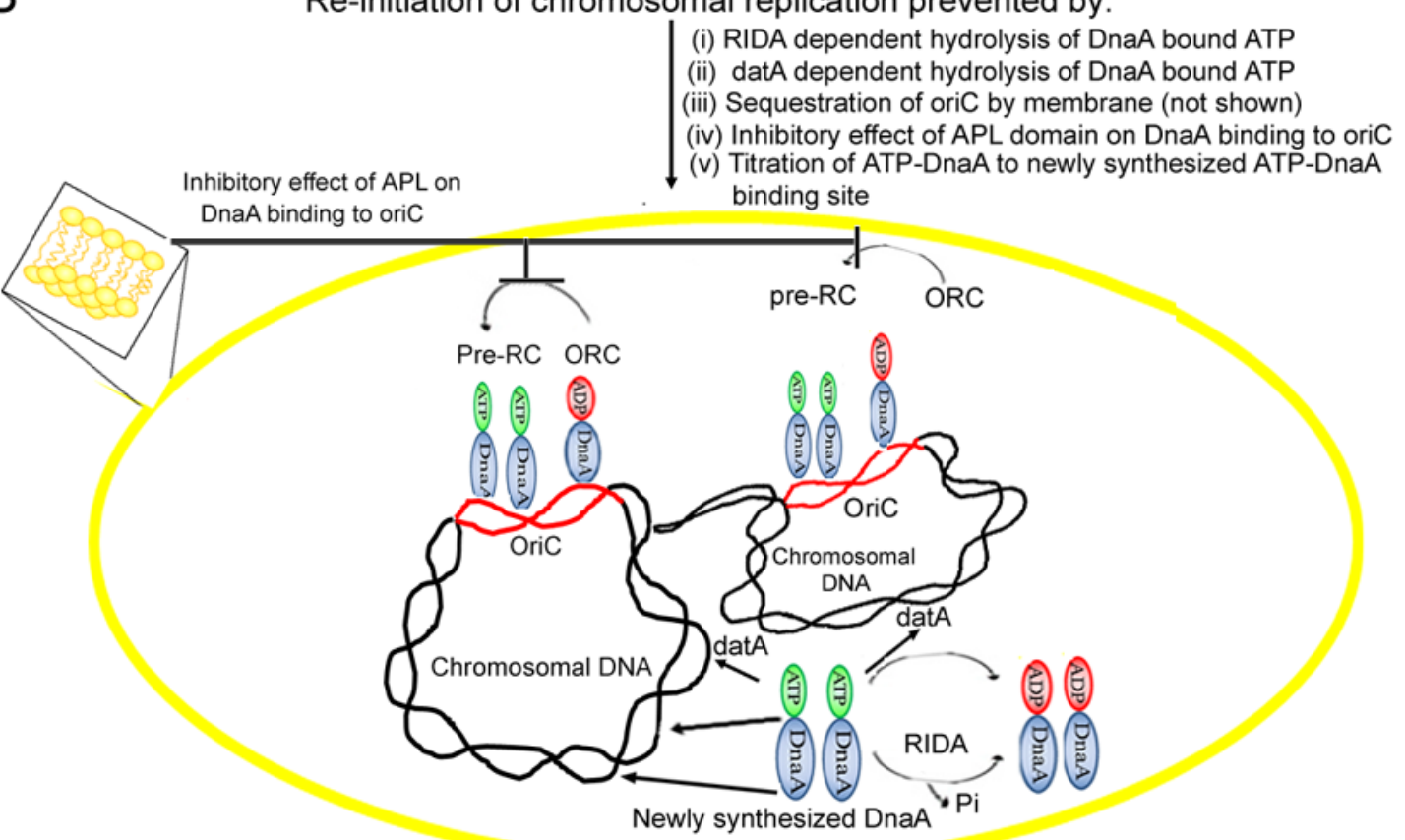


Membrane-catalyzed nucleotide dissociation from DnaA protein is regulated by the DnaA-to-phospholipid ratio present on the membrane. In fact, two different functional states of DnaA protein exists at high and low membrane occupancy, which influences the release of nucleotide from protein [98]. Using the fluorescent ATP derivative MANT-ATP, it has been shown that crowding of DnaA protein on membrane could be induced by changes in temperature or the presence of Ficoll as a crowding agent [98].

The role of specific intergenic sequences, known as DnaA reactivating sequences, or DARS, has also been shown to promote rejuvenation of ADP-DnaA to ATP-DnaA, independent of CL [99,100]. However, cells deficient in acidic phospholipids, but possessing DARS are not able to grow [39-41]. Thus, DARS may not be the predominant or sole mechanism to carry out DnaA rejuvenation in the bacterial cells.

\section{Membrane Fluidity Determines the Rate of ADP to ATP-DnaA Exchange}

In addition to membrane lipids needing an acidic head group to promote the rejuvenation of DnaA, the bilayer must also be in the fluid phase. The fatty acid components of PG-containing small unilamellar vesicles has a strong impact on whether the vesicles are effective at releasing ADP from DnaA, with dipalmitoyl-PG approximately 10-fold less active than dioleoyl-PG [47,48]. Furthermore, phospholipids isolated from the bacteria lacking unsaturated fatty acids are feeble at promoting the exchange of DnaA-bound nucleotide $[47,48]$. This was seen by extracting lipids from E. coli treated with 3-decynoyl- $N$-acetylcysteamine (DNAC), an analog that interrupts the synthesis of unsaturated fatty acids and prevents the initiation of replication in vivo [101]. However, addition of oleic acid to the growth medium relieves the adverse effect of DNAC on growth, and phospholipids extracted from oleic acid-rescued DNAC-treated cells are active at promoting the rejuvenation of ADP-DnaA protein $[47,48]$. Moreover, phospholipids extracted from $f a b A$ mutant cells, which are defective in the synthesis of unsaturated fatty acids [101], grown in absence or presence of oleic acid vary significantly with respect to their fluidity and ability to dissociate DnaA-bound ADP [47,48]. Indeed, a tight correlation was observed between the degree of membrane fluidity, as measured by fluorescence anisotropy, and the extent that the membranes can stimulate nucleotide release from DnaA $[47,48]$.

$E$. coli vary the fatty acid composition of membrane phospholipids with changes in temperature, thereby allowing the bacteria to modulate membrane fluidity in order to optimize cellular functions at different temperatures [102,103]. Suggestive that DnaA-mediated initiation of chromosomal replication is a function affected by membrane fluidity are observations that levels of unsaturated fatty acids are lower in cells harboring a dnaA temperature-sensitive allele than in wild-type cells at elevated temperatures [104], and conversely, the levels of unsaturated fatty acids are less in cells with a cold-sensitive dnaA allele that causes hyperinitiation than in wild-type cells at lower temperatures [104,105]. These changes in fatty acid composition have been proposed help stimulate the feeble initiation activity of the temperature-sensitive DnaA protein at higher temperatures and restrain the hyperinitiation activity of the cold-sensitive DnaA protein at lower temperatures, and that the changes in fatty acid composition occur through DnaA transcriptionally regulating the expression of proteins involved in fatty acid metabolism [104]. 


\section{A Discrete Region of DnaA Associates with Fluid Bilayers Possessing Acidic Phospholipids}

Immunofluorescence microscopy and immunogold labeling of cryothin sections with affinity purified anti-DnaA protein revealed that the majority of DnaA in a cell is localized at the plasma membrane, with approximately a 35-fold higher density in close proximity to the cell membrane than in the cytosol [61].

Two independent studies indicate that DnaA has a specific region that is responsible for its interaction with acidic membranes. In the first study, limited proteolytic digestion of DnaA with chymotrypsin and trypsin generated fragments of $35 \mathrm{kDa}$ (residues D118-F458 of DnaA) and $29 \mathrm{kDa}$ (residues S115-K372 of DnaA), respectively. Both fragments retained high-affinity for adenine nucleotides, yet only the larger chymotryptic fragment released bound nucleotide in response to treatment with acidic phospholipids in a fluid bilayer. Moreover, if DnaA was first allowed to associate with acidic membranes before treatment with trypsin, cleavage at K372 to generate the $29 \mathrm{kDa}$ fragment was prevented, and instead a $30 \mathrm{kDa}$ fragment (S115-K381) was obtained. The $30 \mathrm{kDa}$ fragment, like the $35 \mathrm{kDa}$ chymotryptic fragment and full-length DnaA, released its bound nucleotide when incubated with acidic phospholipids at a temperature that bestowed fluidity to the membrane bilayer. Thus, it is likely that the portion of DnaA near lysine 372 directly associates with acidic phospholipid bilayers [106].

Independently, crosslinking studies that utilized the photoactivable phospholipid analog 1-O-hexadecanoyl-2-O-[9-[2-[ $\left.{ }^{125} \mathrm{I}\right]$ iodo-4-(trifluoromethyl-3H-diazirin-3-yl)benzyl] oxy]carbonyl] nonanoyl]-sn-glycero-3-phosphocholine as a probe in acidic and neutral phospholipid bilayers provides additional evidence of a direct interaction between DnaA and acidic phospholipids. The study revealed that DnaA at the site of a putative amphipathic helix (amino acids 354-372) inserts into the hydrophobic interior of lipid bilayers only when the bilayer is enriched in acidic phospholipids and has the same degree of fluidity that promotes nucleotide exchange [107].

\section{DnaA, Acidic Phospholipids, and Electrostatic Interactions}

The requirement for the fluid bilayer to also have acidic headgroups appears to be due to the acidic head groups' participation in the electrostatic recruitment of DnaA, a basic protein, to the lipid bilayer [20]. Such a mechanism is in agreement with the observation that even though E. coli lacks phosphatidylinositol (PI) or sphingolipids, negatively charged PI and ganglioside $\mathrm{GM}_{1}$ (monosialotetrahexosylganglioside) are equal to $\mathrm{PG}$ in their capacity to stimulate the release of DnaA-bound adenine nucleotide [46,106]. Notably, PG, PI and ganglioside $\mathrm{GM}_{1}$ have structurally distinct polar head groups. Furthermore, in contrast to ganglioside $\mathrm{GM}_{1}$, asialo- $\mathrm{GM}_{1}$ is feeble at promoting nucleotide exchange [106]. Perhaps not surprising, CL with its more anionic nature is significantly more potent in reactivating DnaA when compared to other acidic glycerophospholipids [46,106]. Phosphatadic acid (PA), another anionic phospholipid can also stimulate the exchange ADP to ATP over DnaA protein $[48,106]$. Interestingly, cells with little PG and CL have significantly higher levels of PA [65]. Together, these observations suggest that fluid bilayer's enriched with acidic head groups is more important than any specific head group structure in promoting membrane-mediated ADP-ATP exchange on DnaA. 
Supporting the importance of electrostatic forces for DnaA-membrane association, stable DnaA-lipid bilayer interaction is sensitive to ionic strength, as assessed by isopycnic centrifugation and intrinsic tryptophan fluorescence measurements [20]. Site-directed mutation analysis of DnaA structure-function revealed that basic residues Arg-360, Arg-364 and Lys-372 are indispensable for CL-mediated release of DnaA-bound nucleotide [108,109]. Of note, the region of DnaA (residues Asp-357-Val-374) containing these key residues is the same as that containing the proposed amphipathic helix involved in membrane binding of DnaA protein [106,107]. This prediction of an amphipathic helix in E. coli DnaA is supported by the solved crystal structure for a truncated form of Aquifex aeolicus DnaA [110]. Indeed, sequence comparisons show that these amino acids are well conserved among different bacterial species [110].

Examinations of additional point mutations revealed that the amino acids Arg-328, Arg-334 and Arg-342 present in another potential amphipathic helix (Lysine-327-Ile-345) also are important for DnaA-CL association [111]. Of these, Arg-328 and Lys-372 seems to be the most critical since CL interactions with these basic amino acid residues may change the confirmation of the ATP binding pocket, which further stimulates the release of ADP from the protein [112,113].

\section{Acidic Phospholipids Inhibit DnaA Binding to $E$. coli oriC DNA}

In addition to promoting exchange of DnaA-bound nucleotide, CL has been proposed to also inhibit DnaA binding to oriC (Figure 4). Filter retention assays demonstrated that nucleoprotein complexes of DnaA-oriC DNA remain intact when treated with PG or CL and ATP [49]. However, when nucleotide-bound or nucleotide-free DnaA is first treated with anionic phospholipids, the DnaA no longer is able to bind oriC DNA [49]. Thus, reactivation of ADP-DnaA to ATP-DnaA only occurs when DnaA, oriC, ATP, and anionic phospholipids in a fluid bilayer act in concert.

Interestingly, the degree that different phospholipids inhibit DnaA binding to oriC follows in a similar order to that observed for nucleotide exchange. CL was found to be the most potent in inhibiting DnaA-oriC interaction, whereas PG showed a 10-fold less inhibitory effect on the assembly of DnaA at oriC. Treating DnaA with neutral lipids, such as PC and PE, had little consequences for DnaA binding to oriC [50]. As with nucleotide exchange, the physical state of the bilayer influences the capacity of acidic phospholipids to inhibit DnaA-oriC interaction. Vesicles composed of di-linoleoyl PG inhibit the formation of DnaA-oriC nucleoprotein complexes more effectively than PG-liposomes with stearic acid acyl components [50].

Recently we observed that nucleotide-free DnaA protein exposed to liposomes of dilinoleoyl PG, $\mathrm{CL}$ or a mixture of phospholipids extracted from exponentially growing E. coli cultures abolishes DnaA binding to both high and low affinity DnaA sequences within oriC. On the other hand, nucleoprotein complexes formed in the presence of ATP or ADP remains unaffected by subsequent addition of purified acidic phospholipids as well as total lipids extracted from E. coli (unpublished result). These observations reflect the possibility that the ordered assembly of DnaA protein at specific recognition sequences might depend on whether DnaA is first exposed to cognate nucleic acid binding sites or to acidic phospholipids. Notably, the negatively charged polar head groups of acidic phospholipids and the negatively charged phosphodiester backbone of DNA may interact in a similar manner with basic proteins (Figure 4) [114,115], such as to preclude DnaA from binding to oriC when 
bound to the acidic phospholipids. If such negative control regulates DnaA binding to oriC to prevent re-initiation (Figure 4), it is unknown how this negative effect on DnaA activity is relieved or bypassed to allow normal initiations at the proper time in the cell-cycle. Additionally, the possibility cannot be excluded that cellular levels of acidic phospholipids might affect the binding of DnaA protein to cognate sequences other than oriC, such as, DARS and datA, due to similar nature DNA-binding interactions.

Studies have shown that total lipids isolated from exponentially growing Staphylococcus aureus are active in promoting the release of bound nucleotide from $S$. aureus DnaA protein, whereas lipid isolated from cells in stationary phase were inactive [116]. Of interest, earlier studies found that cellular lipid composition varies with the growth phase of $E$. coli, with a significant increase in CL levels as cells enter into stationary phase [51,52], perhaps in contrast to what one would expect for those lipids being active at promoting nucleotide release. Conversely, an increase of CL in stationary phase could be commensurate with the observed function of CL in inhibiting DnaA binding to oriC. Moreover, changes in lipid composition as cells move from one growth phase to another may differ between bacterial species.

\section{Cardiolipin Helps Sub-Cellular Localization of Certain Bacterial Proteins}

Acidic phospholipids, rather being homogenously distributed over the surface of bacterial cell membrane, exist as discrete domains at the poles and in the septal region of the cytoplasmic membrane of bacteria such as E. coli [12] and B. subtilis [44]. These domains-like structures can be visualized in living cells using the CL-specific fluorescent dye, $N A O[12,15,44]$. Unpublished observations suggest that the number and the location of CL-enriched domains in E. coli change as cells progress through the cell cycle.

E. coli having mutations in cytoplasmic division proteins form miniature cells (or mini cells) that lack DNA as a result of cell division occurring near the cell pole [117,118]. Examination of the membrane from minicells in E. coli [118] and forespore membranes in B. subtilis [44] produced during sporulation, showed enhanced levels of CL, which form domain-like structures.

The presence of certain lipids at regions of membrane curvature serve to target protein-lipid complexes to cell poles $[53,56,58,119]$. The cell division protein MinD that acts to inhibit septum Z-ring formation [120] preferentially binds to CL and localizes to the negatively curved regions of E. coli membranes [53]. CL is also shown to promote the polar location of other proteins besides MinD, such as E. coli osmosensory transporter ProP [56-58], and mechanosensitive channel protein MscS [58] and DivIVA in B. subtilis [121].

Whereas some protein are found concentrated at poles, there are proteins that form helices beneath the cell membrane, extending from pole to pole along the cell's longitudinal axis, such as the cytoskeleton protein MreB and MinCDE [122,123]. Although E. coli DnaA, visualized by confocal microscopy of an internally-tagged GFP-DnaA fusion, also forms helical structures along the longitudinal cell axis, these helical structures exist distinct from and independent of MreB filaments [62]. Other studies using a chromosomally-encoded DnaA-EYFP protein did not detect a helical structure for DnaA [124], but this tagged protein was proposed [124] to be more active in initiation than the internally-tagged GFP-DnaA fusion, suggesting differences between the two proteins. Moreover, 
problems with photobleaching prevented visualization of the DnaA-EYFP protein by confocal microscopy [124]. A plasmid-born mcherry-tagged DnaA protein did not reveal helical structures, but that is not surprising given the high level of overexpression of mcherry-DnaA [125]. Interestingly, the overall global helical pattern formed by internally-tagged GFP-DnaA protein remains unaltered in the bacteria containing significantly reduced levels of acidic phospholipids (unpublished results), suggesting that localization of DnaA protein is not influenced by CL domains within the cell. Mechanisms that serve to regulate the spatial distribution of DnaA remain unknown.

\section{Concluding Remarks}

There is a wealth of data suggesting that the ability of DnaA to normally initiate E. coli chromosomal replication at oriC is influenced by acidic phospholipids present in the cell membrane. Depletion of the acidic phospholipids via repressed expression of pgsA results in under initiation of replication and arrested cell growth. The growth arrest phenotype can be relieved by abnormal initiations events at loci other than oriC or by expression of DnaA protein harboring a point mutation in its membrane-binding, both of which suggest a close link between cellular membrane composition and essential DnaA-mediated initiations at oriC. Of interest, preventing the accumulation at inner membrane of an intermediate of the murein lipoprotein (Lpp) synthesis pathway also can relieve the growth-arrest phenotype of acidic phospholipid-deficient cells, raising the question of whether the accumulated intermediate of Lpp synthesis adversely affects DnaA's action at oriC.

Acidic phospholipids are concentrated in domain-like structures within the bacterial cell membrane, which change in a cell-cycle dependent manner. The acidic phospholipids may help determine the sub-cellular localization of proteins involved in cell division. It is worth asking might these anionic phospholipid domains also help dictate functional subcellular localization of DnaA protein bound to oriC, and are changes that occur to these domains during the cell-cycle somehow linked to the onset of replication of chromosomal DNA?

Acidic phospholipids PG and CL may participate in multiple critical cellular processes related to chromosomal replication. These include: (i) rejuvenation of ADP-DnaA to ATP-DnaA to support rounds of replication in subsequent cell-cycles; (ii) inhibition of DnaA binding to oriC to help set the precise timing of when DNA synthesis occurs; and (iii) possibly helping define the subcellular localization of chromosomal replication components. As such, we hypothesize crosstalk between DnaA protein and acidic phospholipids.

\section{Acknowlegements}

This work was supported in part by the Georgetown University Medical Center Office for the Dean for Research.

\section{Conflict of Interest}

The authors declare no conflict of interest. 


\section{References}

1. Raetz, C.R. Molecular genetics of membrane phospholipid synthesis. Annu. Rev. Genet. 1986, 20, 253-295.

2. Kanfer, J.N.; Kennedy, E.P. Synthesis of phosphatidylserine by Escherichia coli. J. Biol. Chem. 1962, 237, 270-271.

3. Kanfer, J.N.; Kennedy, E.P. Metabolism and function of bacterial lipids. ii. Biosynthesis of phospholipids in Escherichia coli. J. Biol. Chem. 1964, 239, 1720-1726.

4. Raetz, C.R.; Dowhan, W. Biosynthesis and function of phospholipids in Escherichia coli. J. Biol. Chem. 1990, 265, 1235-1238.

5. Cronan, J.E. Bacterial membrane lipids: Where do we stand? Annu. Rev. Microbiol. 2003, 57, 203-224.

6. Ohta, A.; Obara, T.; Asami, Y.; Shibuya, I. Molecular cloning of the cls gene responsible for cardiolipin synthesis in Escherichia coli and phenotypic consequences of its amplification. J. Bacteriol. 1985, 163, 506-514.

7. Nishijima, S.; Asami, Y.; Uetake, N.; Yamagoe, S.; Ohta, A.; Shibuya, I. Disruption of the Escherichia coli cls gene responsible for cardiolipin synthesis. J. Bacteriol. 1988, 170, 775-780.

8. Tropp, B.E. Cardiolipin synthase from Escherichia coli. Biochim. Biophys. Acta 1997, 1348, 192-200.

9. Depalo, N.; Catucci, L.; Mallardi, A.; Corcelli, A.; Agostiano, A. Enrichment of cardiolipin content throughout the purification procedure of photosystem II. Bioelectrochemistry 2004, 63, 103-106.

10. Ventrella, A.; Catucci, L.; Mascolo, G.; Corcelli, A.; Agostiano, A. Isolation and characterization of lipids strictly associated to PSII complexes: Focus on cardiolipin structural and functional role. Biochim. Biophys. Acta 2007, 1768, 1620-1627.

11. Laczkó-Dobos, H.; Ughy, B.; Tóth, S.Z.; Komenda, J.; Zsiros, O.; Domonkos, I.; Párducz, A.; Bogos, B.; Komura, M.; Itoh, S.; et al. Role of phosphatidylglycerol in the function and assembly of Photosystem II reaction center, studied in a cdsA-inactivated PAL mutant strain of Synechocystis sp. PCC6803 that lacks phycobilisomes. Biochim. Biophys. Acta 2008, 1777, 1184-1194.

12. Mileykovskaya, E.; Dowhan, W. Visualization of phospholipid domains in Escherichia coli by using the cardiolipin-specific fluorescent dye 10-N-nonyl acridine orange. J. Bacteriol. 2000, 182, 1172-1175.

13. Matsumoto, K.; Kusaka, J.; Nishibori, A.; Hara, H. Lipid domains in bacterial membranes. Mol. Microbiol. 2006, 61, 1110-1117.

14. Schlame, M. Cardiolipin synthesis for the assembly of bacterial and mitochondrial membranes. J. Lipid. Res. 2008, 49, 1607-1620.

15. Mileykovskaya, E.; Dowhan, W. Cardiolipin membrane domains in prokaryotes and eukaryotes. Biochim. Biophys. Acta 2009, 1788, 2084-2091.

16. Daum, G. Lipids of mitochondria. Biochim. Biophys. Acta 1985, 822, 1-42.

17. Schlame, M.; Haldar, D. Cardiolipin is synthesized on the matrix side of the inner membrane in rat liver mitochondria. J. Biol. Chem. 1993, 268, 74-79. 
18. De Andrade Rosa, I.; Einicker-Lamas, M.; Bernardo, R.R.; Previatto, L.M.; Mohana-Borges, R.; Morgado-Díaz, J.A.; Benchimol, M. Cardiolipin in hydrogenosomes: Evidence of symbiotic origin. Eukaryot. Cell 2006, 5, 784-787.

19. Lensink, M.F.; Govaerts, C.; Ruysschaert, J.M. Identification of specific lipid-binding sites in integral membrane proteins. J. Biol. Chem. 2010, 285, 10519-10526.

20. Kitchen, J.L.; Li, Z.; Crooke, E. Electrostatic interactions during acidic phospholipid reactivation of DnaA protein, the Escherichia coli initiator of chromosomal replication. Biochemistry 1999, $38,6213-6221$.

21. Murray, D.; Arbuzova, A.; Honig, B.; McLaughlin, S. The role of electrostatic and nonpolar interactions in the association of peripheral proteins with membranes Pept. Lipid Interact. 2002, 52, 277-307.

22. Mulgrew-Nesbitt, A.; Diraviyam, K.; Wang, J.Y.; Singh, S.; Murray, P.; Li, Z.H.; Rogers, L.; Mirkovic, N.; Murray, D. The role of electrostatics in protein-membrane interactions. Biochim. Biophys. Acta 2006, 1761, 812-826.

23. Vorobyov, I.; Allen, T.W. On the role of anionic lipids in charged protein interactions with membranes. Biochim. Biophys. Acta 2011, 1808, 1673-1683.

24. McMillin, J.B.; Dowhan, W. Cardiolipin and apoptosis. Biochim. Biophys. Acta 2002, 1585, 97-107.

25. Gonzalvez, F.; Gottlieb, E. Cardiolipin: Setting the beat of apoptosis. Apoptosis 2007, 12, 877-885.

26. Schug, Z.T.; Gottlieb, E. Cardiolipin acts as a mitochondrial signalling platform to launch apoptosis. Biochim. Biophys. Acta 2009, 1788, 2022-2031.

27. Haines, T.H.; Dencher, N.A. Cardiolipin: A proton trap for oxidative phosphorylation. FEBS Lett. 2002, 528, 35-39.

28. Gohil, V.M.; Hayes, P.; Matsuyama, S.; Schägger, H.; Schlame, M.; Greenberg, M.L. Cardiolipin biosynthesis and mitochondrial respiratory chain function are interdependent. J. Biol. Chem. 2004, 279, 42612-42618.

29. Arias-Cartin, R.; Grimaldi, S.; Pommier, J.; Lanciano, P.; Schaefer, C.; Arnoux, P.; Giordano, G.; Guigliarelli, B.; Magalon, A. Cardiolipin-based respiratory complex activation in bacteria. Proc. Natl. Acad. Sci.USA 2011, 108, 7781-7786.

30. Arias-Cartin, R.; Grimaldi, S.; Arnoux, P.; Guigliarelli, B.; Magalon A. Cardiolipin binding in bacterial respiratory complexes: Structural and functional implications. Biochim. Biophys. Acta 2012, 1817, 1937-1949.

31. Wenz, T.; Hielscher, R.; Hellwig, P.; Schägger, H.; Richers, S.; Hunte, C. Role of phospholipids in respiratory cytochrome bc(1) complex catalysis and supercomplex formation. Biochim. Biophys. Acta 2009, 1787, 609-616.

32. Laskowska, E.; Kuczyńska-Wiśnik, D.; Skórko-Glonek, J.; Taylor, A. Degradation by proteases Lon, Clp and HtrA, of Escherichia coli proteins aggregated in vivo by heat shock; HtrA protease action in vivo and in vitro. Mol. Microbiol. 1996, 22, 555-571.

33. Rosen, R.; Biran, D.; Gur, E.; Becher, D.; Hecker, M.; Ron, E.Z. Protein aggregation in Escherichia coli: Role of proteases. FEMS Microbiol. Lett. 2002, 207, 9-12. 
34. Minami, N.; Yasuda, T.; Ishii, Y.; Fujimori, K.; Amano, F. Regulatory role of cardiolipin in the activity of an ATP-dependent protease, Lon, from Escherichia coli. J. Biochem. 2011, 149, 519-527.

35. Mileykovskaya, E.; Dowhan, W. Role of membrane lipids in bacterial division-site selection. Curr. Opin. Microbiol. 2005, 8, 135-142.

36. Xia, W.; Dowhan, W. In vivo evidence for the involvement of anionic phospholipids in initiation of DNA replication in Escherichia coli. Proc. Natl. Acad. Sci. USA 1995, 92, 783-787.

37. Zheng, W.; Li, Z.; Skarstad, K.; Crooke, E. Mutations in DnaA protein suppress the growth arrest of acidic phospholipid-deficient Escherichia coli cells. EMBO J. 2001, 20, 1164-1172.

38. Boeneman, K.; Crooke, E. Chromosomal replication and the cell membrane. Curr. Opin. Microbiol. 2005, 8, 143-148.

39. Fingland, N.; Flåtten, I.; Downey, C.D.; Fossum-Raunehaug, S.; Skarstad, K.; Crooke, E. Depletion of acidic phospholipids influences chromosomal replication in Escherichia coli. Microbiologyopen 2012, 1, 450-466.

40. Heacock, P.N.; Dowhan, W. Construction of a lethal mutation in the synthesis of the major acidic phospholipids of Escherichia coli. J. Biol. Chem. 1987, 262, 13044-13049.

41. Heacock, P.N.; Dowhan, W. Alteration of the phospholipid composition of Escherichia coli through genetic manipulation. J. Biol. Chem. 1989, 264, 14972-14977.

42. Pluschke, G.; Hirota, Y.; Overath, P. Function of phospholipids in Escherichia coli. Characterization of a mutant deficient in cardiolipin synthesis. J. Biol. Chem. 1978, 253, 5048-5055.

43. Tan, B.K.; Bogdanov, M.; Zhao, J.; Dowhan, W.; Raetz, C.R.; Guan, Z. Discovery of a cardiolipin synthase utilizing phosphatidylethanolamine and phosphatidylglycerol as substrates. Proc. Natl. Acad. Sci. USA 2012, 109, 16504-16509.

44. Kawai, F.; Shoda, M.; Harashima, R.; Sadaie, Y.; Hara, H.; Matsumoto, K. Cardiolipin domains in Bacillus subtilis marburg membranes. J. Bacteriol. 2004, 186, 1475-1483.

45. Guo, D.; Tropp, B.E. A second Escherichia coli protein with CL synthase activity. Biochim. Biophys. Acta 2000, 1483, 263-274.

46. Sekimizu, K.; Kornberg, A. Cardiolipin activation of dnaA protein, the initiation protein of replication in Escherichia coli. J. Biol. Chem. 1988, 263, 7131-7135.

47. Yung, B.Y.; Kornberg, A. Membrane attachment activates dnaA protein, the initiation protein of chromosome replication in Escherichia coli. Proc. Natl. Acad. Sci. USA 1988, 85, 7202-7205.

48. Castuma, C.E.; Crooke, E.; Kornberg, A. Fluid membranes with acidic domains activate DnaA, the initiator protein of replication in Escherichia coli. J. Biol. Chem.1993, 268, 24665-24668.

49. Crooke, E.; Castuma, C.E.; Kornberg, A. The chromosome origin of Escherichia coli stabilizes DnaA protein during rejuvenation by phospholipids. J. Biol. Chem. 1992, 267, 16779-16782.

50. Makise, M.; Mima, S.; Katsu, T.; Tsuchiya, T.; Mizushima, T. Acidic phospholipids inhibit the DNA-binding activity of DnaA protein, the initiator of chromosomal DNA replication in Escherichia coli. Mol. Microbiol. 2002, 46, 245-256.

51. Mozharov, A.D.; Shchipakin, V.N.; Fishov, I.L.; Evtodienko, Yu.V. Changes in the composition of membrane phospholipids during the cell cycle of Escherichia coli. FEBS Lett. 1985, 186, $103-106$. 
52. Hiraoka, S.; Matsuzaki, H.; Shibuya, I. Active increase in cardiolipin synthesis in the stationary growth phase and its physiological significance in Escherichia coli. FEBS Lett. 1993, 336, 221-224.

53. Renner, L.D.; Weibel, D.B. Cardiolipin microdomains localize to negatively curved regions of Escherichia coli membranes. Proc. Natl. Acad. Sci. USA 2011, 108, 6264-6269.

54. Szeto, T.H.; Rowland, S.L.; Rothfield, L.I.; King, G.F. Membrane localization of MinD is mediated by a $C$-terminal motif that is conserved across eubacteria, archaea, and chloroplasts. Proc. Natl. Acad. Sci. USA 2002, 99, 15693-15698.

55. Mileykovskaya, E.; Fishov, I.; Fu, X.; Corbin, B.D.; Margolin, W.; Dowhan, W. Effects of phospholipid composition on MinD-membrane interactions in vitro and in vivo. J. Biol. Chem. 2003, 278, 22193-22198.

56. Romantsov, T.; Helbig, S.; Culham, D.E.; Gill, C.; Stalker, L.; Wood, J.M. Cardiolipin promotes polar localization of osmosensory transporter ProP in Escherichia coli. Mol. Microbiol. 2007, 64, 1455-1465.

57. Mileykovskaya, E. Subcellular localization of Escherichia coli osmosensory transporter ProP: Focus on cardiolipin membrane domains. Mol. Microbiol. 2007, 64, 1419-1422.

58. Romantsov, T.; Battle, A.R.; Hendel, J.L.; Martinac, B.; Wood, J.M. Protein localization in Escherichia coli cells: Comparison of the cytoplasmic membrane proteins ProP, LacY, ProW, AqpZ, MscS, and MscL. J. Bacteriol. 2010, 192, 912-924.

59. Breyton, C.; Haase, W.; Rapoport, T.A.; Kühlbrandt, W. Collinson I. Three-dimensional structure of the bacterial protein-translocation complex SecYEG. Nature 2002, 418, 662-665.

60. Gold, V.A.; Robson, A.; Bao, H.; Romantsov, T.; Duong, F.; Collinson, I. The action of cardiolipin on the bacterial translocon. Proc. Natl. Acad. Sci. USA 2010, 107, 10044-10049.

61. Newman, G.; Crooke, E. DnaA, the initiator of Escherichia coli chromosomal replication, is located at the cell membrane. J. Bacteriol. 2000, 182, 2604-2610.

62. Boeneman, K.; Fossum, S.; Yang, Y.; Fingland, N.; Skarstad, K.; Crooke, E. Escherichia coli DnaA forms helical structures along the longitudinal cell axis distinct from MreB filaments. Mol. Microbiol. 2009, 72, 645-657.

63. Torrey, T.; Atlung, T.; Kogoma, T. dnaA suppressor (dasF) mutants of Escherichia coli are stable DNA replication (sdrA/rnh) mutants. Mol. Gen. Genet. 1984, 196, 350-355.

64. Von Meyenburg, K.; Boye, E.; Skarstad, K.; Koppes, L.; Kogoma, T. Mode of initiation of constitutive stable DNA replication in RNase H-defective mutants of Escherichia coli K-12. J. Bacteriol. 1987, 169, 2650-2658.

65. Kikuchi, S.; Shibuya, I.; Matsumoto, K. Viability of an Escherichia coli pgsA null mutant lacking detectable phosphatidylglycerol and cardiolipin. J. Bacteriol. 2000, 182, 371-376.

66. Inouye, S.; Franceschini, T.; Sato, M.; Itakura, K.; Inouye, M. Prolipoprotein signal peptidase of Escherichia coli requires a cysteine residue at the cleavage site. EMBO J. 1983, 2, 87-91.

67. Sankaran, K.; Wu, H.C. Lipid modification of bacterial prolipoprotein. Transfer of diacylglyceryl moiety from phosphatidylglycerol. J. Biol. Chem. 1994, 269, 19701-19706.

68. Suzuki, M.; Hara, H.; Matsumoto, K. Envelope disorder of Escherichia coli cells lacking phosphatidylglycerol. J. Bacteriol. 2002, 184, 5418-5425. 
69. Li, Z.; Kitchen, J.L.; Boeneman, K.; Anand, P.; Crooke, E. Restoration of growth to acidic phospholipid-deficient cells by DnaA(L366K) is independent of its capacity for nucleotide binding and exchange and requires DnaA. J. Biol. Chem. 2005, 280, 9796-9801.

70. Saxena, R.; Rozgaja, T.; Grimwade, J.; Crooke, E. Remodeling of nucleoprotein complexes is independent of the nucleotide state of a mutant AAA+ protein. J. Biol. Chem. 2011, 286, 33770-33777.

71. Weigel, C.; Schmidt, A.; Seitz, H.; Tüngler, D.; Welzeck, M.; Messer, W. The N-terminus promotes oligomerization of the Escherichia coli initiator protein DnaA. Mol. Microbiol. 1999, 34, 53-66.

72. Simmons, L.A.; Felczak, M.; Kaguni, J.M. DnaA Protein of Escherichia coli: Oligomerization at the $E$. coli chromosomal origin is required for initiation and involves specific $N$-terminal amino acids. Mol. Microbiol. 2003, 49, 849-858.

73. Felczak, M.M.; Simmons, L.A.; Kaguni, J.M. An essential tryptophan of Escherichia coli DnaA protein functions in oligomerization at the E. coli replication origin. J. Biol. Chem. 2005, 280, 24627-24633.

74. Aranovich, A.; Parola, A.H.; Fishov, I. The reactivation of DnaA(L366K) requires less acidic phospholipids supporting their role in the initiation of chromosome replication in Escherichia coli. FEBS Lett. 2007, 581, 4439-4442.

75. Kimura, M.; Miki, T.; Hiraga, S.; Nagata, T.; Yura, T. Conditionally lethal amber mutations in the dnaA region of the Escherichia coli chromosome that affect chromosome replication. J. Bacteriol. 1979, 140, 825-834.

76. Fuller, R.S.; Funnell, B.E.; Kornberg, A. The dnaA protein complex with the Escherichia coli chromosomal replication origin (oriC) and other DNA sites. Cell 1984, 38, 889-900.

77. Sekimizu, K.; Bramhill, D.; Kornberg, A. ATP activates dnaA protein in initiating replication of plasmids bearing the origin of the Escherichia coli chromosome. Cell 1987, 50, 259-265.

78. Bramhill, D.; Kornberg, A. Duplex opening by dnaA protein at novel sequences in initiation of replication at the origin of the Escherichia coli chromosome. Cell 1988, 52, 743-755.

79. Ozaki, S.; Kawakami, H.; Nakamura, K.; Fujikawa, N.; Kagawa, W.; Park, S.Y.; Yokoyama, S.; Kurumizaka H.; Katayama, T. A common mechanism for the ATP-DnaA-dependent formation of open complexes at the replication origin. J. Biol. Chem. 2008, 283, 8351-8362.

80. Marszalek, J.; Kaguni, J.M. DnaA protein directs the binding of DnaB protein in initiation of DNA replication in Escherichia coli. J. Biol. Chem. 1994, 269, 4883-4890.

81. Sutton, M.D.; Carr, K.M.; Vicente, M.; Kaguni, J.M. Escherichia coli DnaA protein. The $N$-terminal domain and loading of DnaB helicase at the Escherichia coli chromosomal origin. J. Biol. Chem. 1998, 273, 34255-34262.

82. Kaguni, J.M. Replication initiation at the Escherichia coli chromosomal origin. Curr. Opin. Chem. Biol. 2011, 15, 606-613.

83. Matsui, M.; Oka, A.; Takanami, M.; Yasuda, S.; Hirota, Y. Sites of dnaA protein-binding in the replication origin of the Escherichia coli K-12 chromosome. J. Mol. Biol. 1985, 184, 529-533.

84. McGarry, K.C.; Ryan, V.T.; Grimwade, J.E.; Leonard, A.C. Two discriminatory binding sites in the Escherichia coli replication origin are required for DNA strand opening by initiator DnaA-ATP. Proc. Natl. Acad. Sci. USA 2004, 101, 2811-2816. 
85. Kawakami, H.; Keyamura, K.; Katayama, T. Formation of an ATP-DnaA-specific initiation complex requires DnaA Arginine 285, a conserved motif in the AAA+ protein family. J. Biol. Chem. 2005, 280, 27420-27430.

86. Nievera, C.; Torgue, J.J.; Grimwade, J.E.; Leonard, A.C. SeqA blocking of DnaA-oriC interactions ensures staged assembly of the Escherichia coli pre-RC. Mol Cell. 2006, 24, 581-592.

87. Miller, D.T.; Grimwade, J.E.; Betteridge, T.; Rozgaja, T.; Torgue, J.J.; Leonard, A.C. Bacterial origin recognition complexes direct assembly of higher-order DnaA oligomeric structures. Proc. Natl. Acad. Sci. USA 2009, 106, 18479-18484.

88. Cassler, M.R.; Grimwade, J.E.; Leonard, A.C. Cell cycle-specific changes in nucleoprotein complexes at a chromosomal replication origin. EMBO J. 1995, 14, 5833-5841.

89. Katayama, T.; Kubota, T.; Kurokawa, K.; Crooke, E.; Sekimizu, K. The initiator function of DnaA protein is negatively regulated by the sliding clamp of the Escherichia coli chromosomal replicase. Cell 1998, 94, 61-71.

90. Kato, J.; Katayama, T. Hda, a novel DnaA-related protein, regulates the replication cycle in Escherichia coli. EMBO J. 2001, 20, 4253-4262.

91. Nakamura, K.; Katayama, T. Novel essential residues of Hda for interaction with DnaA in the regulatory inactivation of DnaA: Unique roles for Hda AAA Box VI and VII motifs. Mol. Microbiol. 2010, 76, 302-317.

92. Keyamura, K.; Katayama, T. DnaA protein DNA-binding domain binds to Hda protein to promote inter-AAA+ domain interaction involved in regulatory inactivation of DnaA. J. Biol. Chem. 2011, 286, 29336-29346.

93. Camara, J.E.; Breier, A.M.; Brendler, T.; Austin, S.; Cozzarelli, N.R.; Crooke, E. Hda inactivation of DnaA is the predominant mechanism preventing hyperinitiation of Escherichia coli DNA replication. EMBO Rep. 2005, 6, 736-741.

94. Riber, L.; Olsson, J.A.; Jensen, R.B.; Skovgaard, O.; Dasgupta, S.; Marinus, M.G.; Løbner-Olesen, A. Hda-mediated inactivation of the DnaA protein and dnaA gene autoregulation act in concert to ensure homeostatic maintenance of the Escherichia coli chromosome. Genes Dev. 2006, 20, 2121-2134.

95. Katayama, T.; Fujimitsu, K.; Ogawa, T. Multiple pathways regulating DnaA function in Escherichia coli: Distinct roles for DnaA titration by the datA locus and the regulatory inactivation of DnaA. Biochimie 2001, 83, 13-17.

96. Kasho, K.; Katayama, T. DnaA binding locus datA promotes DnaA-ATP hydrolysis to enable cell cycle-coordinated replication initiation. Proc. Natl. Acad. Sci. USA 2013, 110, 936-941.

97. Kurokawa, K.; Nishida, S.; Emoto, A.; Sekimizu, K.; Katayama, T. Replication cycle-coordinated change of the adenine nucleotide-bound forms of DnaA protein in Escherichia coli. EMBO J. 1999, 18, 6642-6652.

98. Aranovich, A.; Gdalevsky, G.Y.; Cohen-Luria, R.; Fishov, I.; Parola, A.H. Membrane-catalyzed nucleotide exchange on DnaA. Effect of surface molecular crowding. J. Biol Chem. 2006, 281, 12526-12534.

99. Fujimitsu, K.; Katayama, T. Reactivation of DnaA by DNA sequence-specific nucleotide exchange in vitro. Biochem. Biophys. Res. Commun. 2004, 322, 411-419. 
100. Fujimitsu, K.; Senriuchi, T.; Katayama, T. Specific genomic sequences of Escherichia coli promote replicational initiation by directly reactivating ADP-DnaA. Genes Dev. 2009, 23, 1221-1233.

101. Fralick, J.A.; Lark, K.G. Evidence for the involvement of unsaturated fatty acids in initiating chromosome replication in Escherichia coli. J. Mol. Biol. 1973, 80, 459-475.

102. Marr, A.G.; Ingraham. J.I. Effect of temperature on the composition of fatty acids in Escherichia coli. J. Bacteriol. 1962, 84, 1260-1267.

103. De Mendoza, D.; Cronan, J.E. Thermal regulation of membrane lipid fluidity in bacteria. Trends. Biochem. Sci. 1983, 8, 49-52.

104. Suzuki, E.; Kondo, T.; Makise, M.; Mima, S.; Sakamoto, K.; Tsuchiya, T.; Mizushima, T. Alteration in the contents of unsaturated fatty acids in dnaA mutants of Escherichia coli. Mol. Microbiol. 1998, 28, 95-102.

105. Katayama, T.; Kornberg, A. Hyperactive initiation of chromosomal replication in vivo and in vitro by a mutant initiator protein, DnaAcos, of Escherichia coli. J. Biol. Chem. 1994, 269, 12698-12703.

106. Garner, J.; Crooke, E. Membrane regulation of the chromosomal replication activity of Escherichia coli DnaA requires a discrete site on the protein. EMBO J. 1996, 15, 3477-3485.

107. Garner, J.; Durrer, P.; Kitchen, J.; Brunner, J.; Crooke, E. Membrane-mediated release of nucleotide from an initiator of chromosomal replication, Escherichia coli DnaA, occurs with insertion of a distinct region of the protein into the lipid bilayer. J. Biol. Chem. 1998, 273, 5167-5173.

108. Hase, M.; Yoshimi, T.; Ishikawa, Y.; Ohba, A.; Guo, L.; Mima, S.; Makise, M.; Yamaguchi, Y.; Tsuchiya, T.; Mizushima, T. Site-directed mutational analysis for the membrane binding of DnaA protein. Identification of amino acids involved in the functional interaction between DnaA protein and acidic phospholipids. J. Biol. Chem. 1998, 273, 28651-28656.

109. Makise, M.; Mima, S.; Tsuchiya, T.; Mizushima, T. Identification of amino acids involved in the functional interaction between DnaA protein and acidic phospholipids. J. Biol. Chem. 2000, 275, 4513-4518.

110. Erzberger, J.P.; Pirruccello, M.M.; Berger, J.M. The structure of bacterial DnaA: Implications for general mechanisms underlying DNA replication initiation. EMBO J. 2002, 21, 4763-4773.

111. Yamaguchi, Y.; Hase, M.; Makise, M.; Mima, S.; Yoshimi, T.; Ishikawa, Y.; Tsuchiya, T.; Mizushima, T. Involvement of Arg-328, Arg-334 and Arg-342 of DnaA protein in the functional interaction with acidic phospholipids. Biochem J. 1999, 340, 433-438.

112. Makise, M.; Mima, S.; Tsuchiya, T.; Mizushima, T. Molecular mechanism for functional interaction between DnaA protein and acidic phospholipids: Identification of important amino acids. J. Biol. Chem. 2001, 276, 7450-7456.

113. Makise, M.; Mima, S.; Koterasawa, M.; Tsuchiya, T.; Mizushima T. Biochemical analysis of DnaA protein with mutations in both Arg328 and Lys372. Biochem J. 2002, 362, 453-458.

114. Rauch, J.; Tannenbaum, H.; Stollar, B.D.; Schwartz, R.S. Monoclonal anti-cardiolipin antibodies bind to DNA. Eur. J. Immunol. 1984, 14, 529-534. 
115. Kumar, S.; Kalsi, J.; Bunting, K.; Ravirajan, C.T.; Latchman, D.S.; Pearl, L.H.; Isenberg, D.A. Fine binding characteristics of human autoantibodies-partial molecular characterization. Mol. Immunol. 2004, 41, 495-510.

116. Ichihashi, N.; Kurokawa, K.; Matsuo, M.; Kaito, C.; Sekimizu, K. Inhibitory effects of basic or neutral phospholipid on acidic phospholipid-mediated dissociation of adenine nucleotide bound to DnaA protein, the initiator of chromosomal DNA replication. J. Biol. Chem. 2003, 278, 28778-28786.

117. Adler, H.I.; Fisher, W.D.; Cohen, A.; Hardigree, A.A. Miniature Escherichia coli cells deficient in DNA. Proc. Natl. Acad. Sci. USA 1967, 57, 321-326.

118. Koppelman, C.M.; Tanneke, D.B.; Duursma, M.C.; Heeren, R.M.; Nanninga, N. Escherichia coli minicell membranes are enriched in cardiolipin. J. Bacteriol. 2001, 183, 6144-6147.

119. Huang, K.C.; Mukhopadhyay, R.; Wingreen, N.S. A curvature-mediated mechanism for localization of lipids to bacterial poles. PLoS Comput. Biol. 2006, 2, 1357-1364.

120. Shiomi, D.; Margolin, W. The $C$-terminal domain of MinC inhibits assembly of the $\mathrm{Z}$ ring in Escherichia coli. J. Bacteriol. 2007, 189, 236-243.

121. Lenarcic, R.; Halbedel, S.; Visser, L.; Shaw, M.; Wu, L.J.; Errington, J.; Marenduzzo, D.; Hamoen, L.W. Localisation of DivIVA by targeting to negatively curved membranes. EMBO J. 2009, 28, 2272-2282.

122. Jones, L.J.; Carballido-López, R.; Errington, J. Control of cell shape in bacteria: Helical, actin-like filaments in Bacillus subtilis. Cell 2001, 104, 913-922.

123. Shih, Y.L.; Le, T.; Rothfield, L. Division site selection in Escherichia coli involves dynamic redistribution of Min proteins within coiled structures that extend between the two cell poles. Proc. Natl. Acad. Sci.USA 2003, 100, 7865-7870.

124. Nozaki, S.; Niki, H.; Ogawa, T. Replication initiator DnaA of Escherichia coli changes its assembly form on the replication origin during the cell cycle. J. Bacteriol. 2009, 191, 4807-4814.

125. Regev, T.; Myers, N.; Zarivach, R.; Fishov, I. Association of the chromosome replication initiator DnaA with the Escherichia coli inner membrane in vivo: Quantity and mode of binding. PLoS One 2012, 7, e36441.

(C) 2013 by the authors; licensee MDPI, Basel, Switzerland. This article is an open access article distributed under the terms and conditions of the Creative Commons Attribution license (http://creativecommons.org/licenses/by/3.0/). 